\title{
USING MOBILE AGENT FOR LOCATION- SPECIFIC DATA RETRIEVAL IN MANET
}

\author{
Kenji Tei ${ }^{1,2}$, Nobukazu Yoshioka $^{2}$, Yoshiaki Fukazawa ${ }^{1}$, and Shinichi Honiden ${ }^{2,3}$ \\ ${ }^{1}$ Waseda University, 3-4-1 Okubo, Shinjuku-ku, Tokyo, 169-8555 Japan; ${ }^{2}$ National Institute \\ of Informatics, 2-1-2 Hitotsubashi, Chiyoda-ku, Tokyo, 101-8430 Japan; ${ }^{3}$ The University of \\ Tokyo, 7-3-1 Hongo, Bunkyo-ku, Tokyo, 113-8656 Japan
}

\begin{abstract}
Location-specific data retrieval is an attractive application in a Mobile Ad-hoc Network (MANET). Simple solution for it is that an observer retrieves the data by geocasting from an observer node, but its overhead highly depends on location of the observer and the designated region. We propose a mobile agent approach. A mobile agent migrates from the observer node to a node in the designated region, retrieves data from there, and summarizes, filters, and compresses the retrieved data, This data is sent back to the observer, when the observer request. Since the data is retrieved by the mobile agent located near the data sources, the data retrieval in the mobile agent approach would involve low overhead, even if the observer is far from the target region or moves around. In the MANET, however, even after the first migration, to stay near data sources, a mobile agent should migrate to another node in response to node movements.. In this paper, we propose the Geographically Bound Mobile Agent (GBMA) which is a mobile agent that migrates to always be located in a designated region. Moreover, to clarify where the GBMA should be located and when the GBMA starts to migrate, we introduce two geographic zones: required zone and expected zone. Compared with the conventional methods with geocast or with a conventional mobile agent, the GBMA with these zones for retrieving locationspecific data can reduce the total number of messages.
\end{abstract}

Keywords: location-specific data retrieval, mobile ad-hoc network, mobile agent

\section{Introduction}

Mobile Ad-hoc Networks (MANETs) consisting of mobile wireless nodes that communicate with each other have been receiving great attention[1]. The MANET without a fixed infrastructure is expected to be effective in post disaster areas where fixed infrastructures have been destroyed[2]. We believe that people under such situation become more voluntary and cooperative than usual, and that they will provide their personal mobile devices, such as PDAs or smart phones, to create and to maintain the MANET used for communicating 


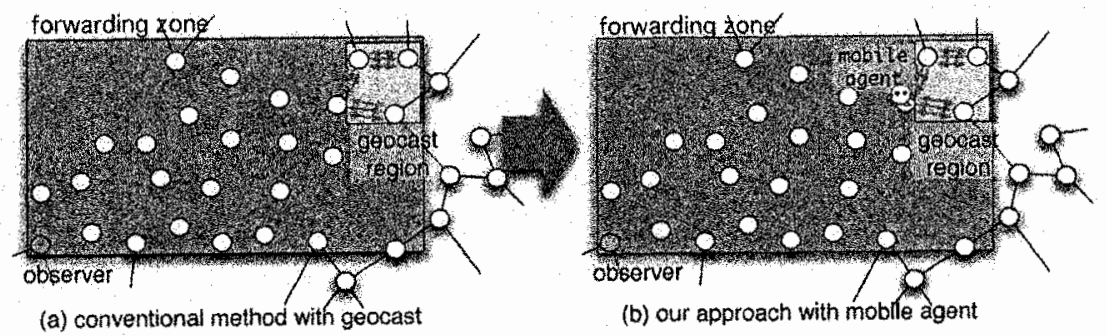

Figure 1. Applying mobile agent in a MANET

with each other. In the post disaster area, location-specific data in particular is exchanged. For example, an observer may confirm a safety of missing people among patients in a certain hospital, locate disaster victims in a certain dangerous place, or inquire what goods are necessary at a certain shelter. We call the region in which the observer is interested designated region in this paper.

Simple approach to the location-specific data retrieval is that an observer sends, from the observer node, messages to retrieve this data. Geocast based on directed flooding[4][5][6] can make this approach produce low message overhead. Geocast[3] is a subclass of multicast with the multicast group defined by a geographic region, and is the delivery of messages to nodes within this region. Geocast based on directed flooding, such as LBM[4], Voronoibased routing[5], or GeoGRID[6], is a subclass of geocast whose messages are delivered by directed flooding. One of major protocols in them is LBM. LBM adopts directed flooding and defines forwarding zone. When receiving a LBM message, only a node in a forwarding zone described in this message forwards this message, and otherwise discards it. Because nodes in the forwarding zone is subset of whole nodes in a MANET, LBM reduces the message flooding overhead. Adopting LBM for the message delivery, the overhead of this approach can be reduced. We call this approach adopting LBM geocast simple geocast approach in this paper.

However, overhead of the simple geocast approach highly depends on the location of an observer and a designated region, even if it adopts LBM. Consider the case that the observer tries to survey the necessary goods at certain shelters. The observer is sometimes located far from the region, and exchanges data from there, as shown in Figure 1 (a). The circles represent nodes and the arrows represent message transfer. The observer (the circle at lower left) frequently interacts with many evacuees in the shelter (the rectangle at top right). Then, data are exchanged many times between the observer and the evacuees. Moreover, one data exchange involves many message forwardings, since forwarding zone covers a large region according to a distance between the ob- 
server and the shelter. As a result, for the observer far from its designated region or moving around, the simple geocast approach will produce the massive number of messages in spite of limiting the number of forwarding nodes.

In this paper, we propose a mobile agent approach[7] for the location-specific data retrieval.In client/server applications, the use of the mobile agent migrating from a client node to a server node and interacting locally with the server can reduce network traffic[8]. We apply this scheme in a MANET. In our method, a mobile agent migrates from an observer node to an evacuee node in a designated region, and retrieves data from evacuee nodes in this region (Figure 1 (b)). After this migration, the mobile agent can retrieve the data via shorter length routes with a smaller number of messages. Therefore, the number of messages involved in the mobile agent approach would not highly depend on location of the observer and the designated region.

However, conventional mobile agent may not provide sufficient solution for the data retrieval. Because each node in a MANET can physically move, the mobile agent host node may move apart from the designated region during the data retrieval. This increases of data retrieval overhead of the mobile agent and makes tracking of the mobile agent difficult. In response to node movements, the mobile agent should migrate among nodes while retrieving data, to remain in the designated region. We propose a mobile agent reactively migrating to remain the designated region in response to the host node movements, and name it Geographically Bound Mobile Agent (GBMA). This GBMA will provide a solution for the location-specific data retrieval.

This section has presented the background of our work. The rest of this paper is organized as follows. Section 2 describes details of the GBMA. Section 3 discusses the effects of applying the GBMA, referring to the simulation results. Section 4 describes related works. Finally, Section 5 presents a conclusion.

\section{Geographically Bound Mobile Agent}

The GBMA is a mobile agent whose location is restricted geographically. GBMA must be hosted on a node located in a designated region, until the tasks are completed. When the current host node of a GBMA leaves the designated region, the GBMA should migrate to another node in the designated region to remain in the region. Restriction of the GBMA location provides two advantages. First, communications between the GBMA and nodes in the region will be relative stable and involve low overhead. Since they communicate via shorter length routes, these communications are hardly prevented by decouplings of MANETs and involve a small amount of messages. Second, locating a GBMA is easy and involves low overhead. Sender needs to discover the GBMA only from nodes in the designated region. 


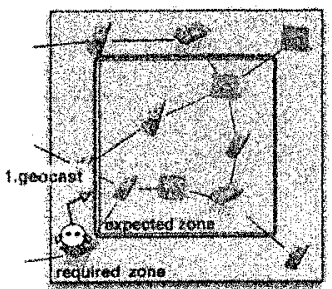

(a)

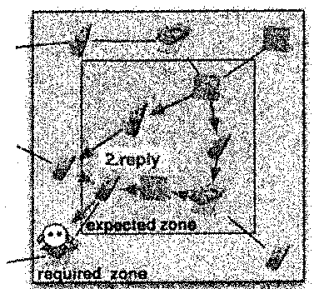

(b)

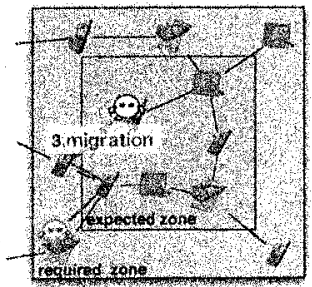

(c)

Figure 2. GBMA migration based on expected zone

However, it is difficult to determine when does start the remigration. The following actions are performed after starting GBMA migration until finishing migration: search for nodes in the designated region and routes to them, select a candidate node based on the aggregated node states, deactivate the GBMA, transfer its program code and data to the candidate node via the discovered route, and reactivate the GBMA on the candidate node. These actions cause migration latency. If a GBMA starts migration after its host node leaves the designated region, it remains outside the designated region until these actions are finished. While it remains outside the designated region, messages sent to it are not received. It must start its migration before its host node leaves the designated region. On the other hand, too early determination of the migration causes needlessly frequent migrations. To reduce the total duration for these migrations, the frequency of its migrations should be low. Therefore, the GBMA must start its migration at an appropriate timing. To define the designated region and to easily adjust the start timing of GBMA migration and the migration frequency, we propose two zones for the GBMA: the required zone and the expected zone.

\subsection{Required zone and expected zone}

A required zone is defined to clarify the region in which the GBMA should be located. The GBMA must be on a node in the required zone. The required zone, which restricts the GBMA location, can be also used when someone sends a message to the GBMA. The sender geocasts with a geocast region represented by the required zone. If the node receiving the message hosts the GBMA, the node passes the message to the GBMA.

On the other hand, an expected zone is defined to clarify the start timing and the frequency of GBMA migration. Figure 2 shows the GBMA actions based on the expected zone. The expected zone must be within the required zone. The GBMA continues to execute its own tasks while it is in the expected zone. When it detects that its host node is outside the expected zone, it starts to 
search for other nodes located in the expected zone and routes to them (Figure 2 (a)), selects one (Figure 2 (b)), and migrates to it through the discovered route (Figure 2 (c)). A start timing and a frequency of GBMA migration can be easily adjusted by modifying the expected zone size.

\subsection{Application example}

We take up data retrieval in a post disaster area described in Section 1, and describe the behavior of the GBMA. An observer surveys goods needed by evacuees in several shelters via a MANET. For each shelters located far from the observer, the observer uses GBMAs to retrieve data about necessary goods.

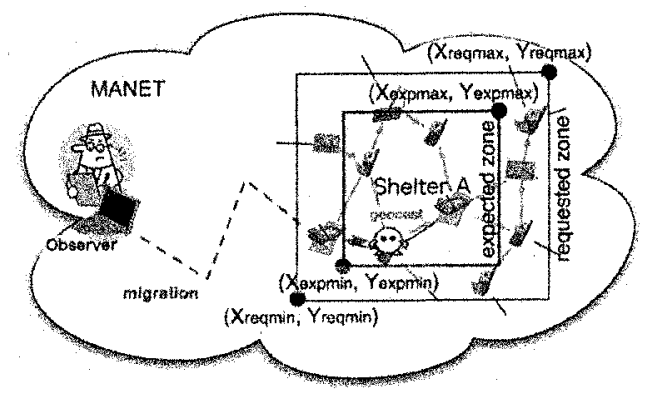

Figure 3. GBMA behavior example

Consider the case illustrated in Figure 3. The observer is interested in shelter A. First, the observer configures a required zone represented by coordinates $\left(x_{\text {reqmin }}, y_{\text {reqmin }}\right)$ and $\left(x_{\text {reqmax }}, y_{\text {reqmax }}\right)$ and an expected zone represented by coordinates $\left(x_{\text {expmin }}, y_{\text {expmin }}\right)$ and $\left(x_{\text {expmax }}, y_{\text {expmax }}\right)$ to the GBMA.

$\left(x_{\text {reqmin }}, y_{\text {reqmin }}\right)$ and $\left(x_{\text {reqmax }}, y_{\text {reqmax }}\right)$ are set to a region that includes the shelter A. $\left(x_{\text {expmin }}, y_{\text {expmin }}\right)$ and $\left(x_{\text {expmax }}, y_{\text {expmax }}\right)$ are set to be within the requested zone: $x_{\text {expmin }} \geq x_{\text {reqmin }}, y_{\text {expmin }} \geq y_{\text {reqmin }}, x_{\text {expmax }} \leq x_{\text {reqmax }}$, and $y_{\text {expmax }} \leq y_{\text {reqmax }}$. Configuring $\left(x_{\text {expmin }}, y_{\text {expmin }}\right)$ and $\left(x_{\text {expmax }}, y_{\text {expmax }}\right)$, the start timing and frequency of GBMA migration can be easily adjusted.

Next, the GBMA on the observer node migrates to a node in the expected zone. GBMA migration protocol somewhat like Location-Aided Routing (LAR) [9] protocol. LAR is a source routing protocol based on DSR protocol[10]. Main difference between them is that a route request (RREQ) message in LAR protocol contains a destination node identifier, location of the destination node, and forwarding zone, and is delivered by directed flooding in the same way adopted in LBM protocol. The LAR RREQ can discover routes to a node which has same identifier contained in this RREQ and which is located in a region contained in this RREQ. After the route discovery, reply 
message is transferred to the observer node along the discovered route. In GBMA migration protocol, RREQ can discover routes to not one node but any nodes in the expected zone described by coordinates $\left(x_{\text {expmin }}, y_{\text {expmin }}\right)$ and $\left(x_{\text {expmax }}, y_{\text {expmax }}\right)$, and these nodes reply its route and its location. After that, the agent selects the node nearest to the coordinate $\left(\frac{x_{\text {expmax }}-x_{\text {expmin }}}{2}\right.$, $\left.\frac{y_{\text {expmax }}-y_{\text {expmin }}}{2}\right)$ from among the received replies, and migrates to this node along the discovered route.

After migration, the GBMA in the requested zone starts to survey for necessary goods data in shelter A. The GBMA geocasts messages containing the required zone and the GBMA identifier, to the required zone. Evacuee nodes that receive this message send their own necessary goods data to the GBMA by LAR unicast, with the required zone and the GBMA identifier contained in the received message sent from the GBMA, each time necessary goods are added or modified. The notification messages are received by the GBMA as long as it is not migrating, at least one path from the notification source to it exists, and it is located in the required zone at the time. While aggregating the data, the GBMA periodically checks its host node location. If the GBMA is outside the expected zone, the GBMA starts to migrate in the same way as described above. The GBMA executes such migration and data retrieval.

The GBMA summarizes or filters aggregated data and compresses them in a manner befitting to this application, to reduce data size. Reduction of the data size reduces overhead of reporting back to the observer. On the other hand, if the GBMA deals with personal data, the GBMA needs to encrypt aggregated data. This application level encryptions prevents malicious host node from picking the data aggregated by the GBMA. If the observer wants to get the result, he or she sends a request to the required zone, and the GBMA receiving the request sends back the retrieved data via a route through which the request comes. Since the observer does not communicate with the GBMA until getting results, data retrieval does not prevent from decoupling of MANETs between the observer and the designated region, and he or she can moves freely.

Note that the expected zone size should be carefully chosen. Appropriately adjusting the size of the expected zone can reduce the duration for which the GBMA is outside the required zone. If the expected zone is too large, the GBMA leaves the required zone until migration is finished. Messages sent to the GBMA while the GBMA is outside the expected zone are lost. If the expected zone is too small, the GBMA frequently migrates and the duration for which the GBMA is migrating increases. The messages to the GBMA, which are sent while the GBMA is migrating are also lost. The GBMA with the optimal size of the expected zone may minimize the number of such lost messages. We examine this issue in Section 3. 


\section{Experiments and discussion}

We evaluate the GBMA using the simulator implemented on JiST/SWANS [11]. Java in Simulation Time (JiST) is a high-performance discrete event simulation engine that runs over a standard Java virtual machine, and Scalable Wireless Ad hoc Network Simulator (SWANS) is a scalable ad hoc network simulator built atop the JiST platform. We implement the LBM protocol and the LAR unicast protocol, and develop the GBMA upon JiST/SWANS. With this simulator, we evaluate two issues concerning the GBMA: the reduction of the number of messages compared with the simple geocast approach with LBM described in Section 1, and the optimal expected zone size.

\subsection{Simulation model}

In the experiments, initially, nodes are distributed according to a grid over a rectangular region of $1000 \mathrm{~m} \times 1000 \mathrm{~m}$ square described by the coordinates $(0,0)$ and $(1000,1000)$. Each node is equipped with an IEEE $802.11 \mathrm{~b}$ wireless device and communicates with other nodes in the range of the wireless device. Each node is also equipped with a GPS device with which it can identify its location. Moreover, each node supports two mobility models: static and random walk[12]. In the static model, each node does not move, and in the random walk model, each node picks a direction randomly, walks a certain distance in that direction, pauses for some time, and repeats. Shelter A is rectangular in shape and defined by the coordinates $(600,600)$ and $(800,800)$.

\subsection{Compared with Simple Geocast Approach}

First, we compare the number of messages produced in a mobile agent approach, to that in the simple geocast approach. In this experiment, let the number of nodes be $12^{2}$ and $14^{2}$. Let the node mobility model be static. Furthermore, let the observer node be located at $(x, x)$ where $\mathrm{x}$ is $600,500,400$, 300,200 , and 100. In the simple geocast approach, the observer retrieves data from nodes in the shelter A. On the other hand, in the mobile agent approach, the observer sends a mobile agent to a node located in shelter A, and the mobile agent on the node retrieves the data. In both approaches, the observer (or the mobile agent) and the evacuees in the shelter $\mathrm{A}$ exchange messages 10 times. The simulation result is depicted in Figure 4.

In Figure 4, dotted lines represent results of the simple geocast approach, and solid lines represent results of the mobile agent approach. In the simple geocast approach, the number of messages increases exponentially, because the number of forwarding nodes increases according to the forwarding zone size. Therefore, the increment rate when the number of nodes is $14^{2}$ is greater than that when the number of nodes is $12^{2}$. On the other hand, in the mobile 


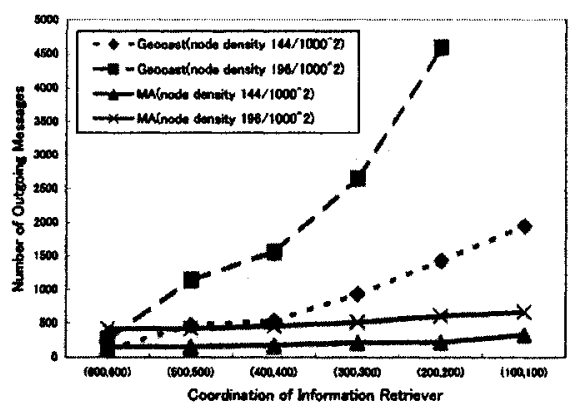

Figure 4. Total number of outgoing messages

agent approach, the increase in the number of the messages is smaller than that in the simple geocast approach. This is because, in the mobile agent approach, a mobile agent exchanges messages via shorter length routes, after migration to shelter A. Therefore, the distance affects the increase in the number of messages only when sending the mobile agent to shelter $\mathrm{A}$, and does not affect message exchanges after migration. When $x$ is 600 , or the observer is located at shelter $\mathrm{A}$, the number of messages in the mobile agent approach is slightly larger than that in the simple geocast approach. This is because, in this case, the data exchange overheads in both approaches are almost the same, but there is the overhead of mobile agent migration in the mobile agent approach. This result shows the mobile agent approach to be effective when the observer is far from the designated region, compared with the simple geocast approach.

\subsection{Optimal expected zone size}

Next, we compare the mobile agent and the GBMA, and examine how the expected zone affects the results. In this experiment, the number of nodes is $15^{2}$ and the node mobility model is the random walk where the node walks $10 \mathrm{~m}$ for 10 seconds and $20 \mathrm{~m}$ for 10 seconds. Initially, the mobile agent or the GBMA is on the node at $(700,700)$; it is located at the center of shelter A. In the mobile agent approach, the mobile agent continues to retrieve data at the initial hosted node in spite of the node movement. In the GBMA approach, the GBMA migrates according to the node movement. The requested zone of GBMA is defined by the coordinates $(600,600)$ and $(800,800)$, and the expected zone is defined by the central coordinate of them and the length on the side being $200,180,160,140,120,100$, or 80 meters. When the length is 200 meters, the expected zone is the same region as the required zone. The smaller the side length is, the smaller the expected zone size is. The GBMA (or the mobile agent) initially geocasts a subscription message, and the nodes that receive it send messages to the GBMA at about one-minute intervals twenty 


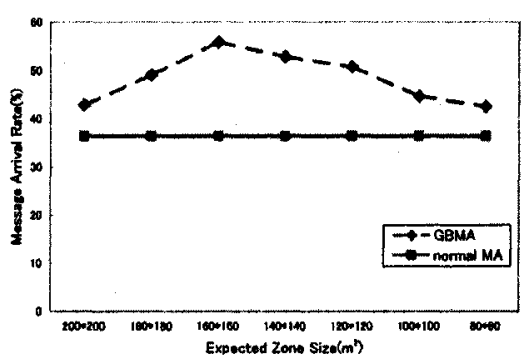

Figure 5. Message arrival rate $(20 \mathrm{~m} / 10 \mathrm{~s})$

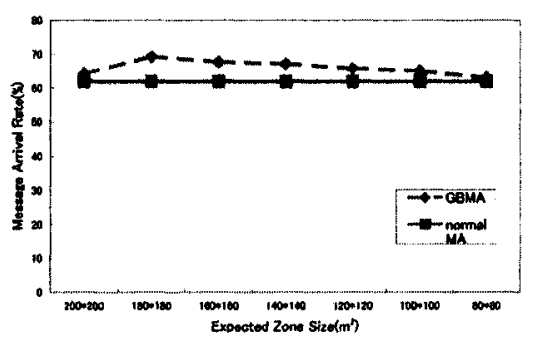

Figure 7. Message arrival rate( $10 \mathrm{~m} / 10 \mathrm{~s})$

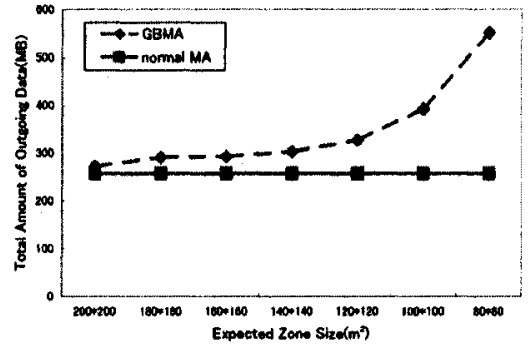

Figure 6. Total amount of outgoing $\operatorname{data}(20 \mathrm{~m} / 10 \mathrm{~s})$

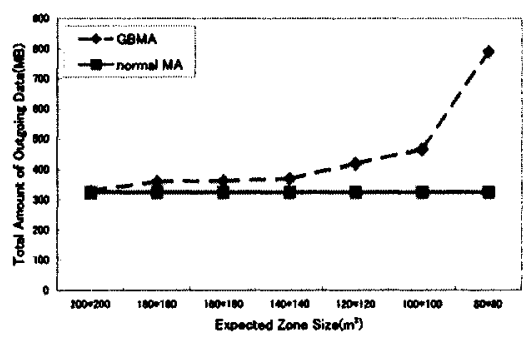

Figure 8 . Total amount of outgoing $\operatorname{data}(10 \mathrm{~m} / 10 \mathrm{~s})$

times. We measure the message arrival rate, which indicates the number of messages received by the GBMA (or the mobile agent) per total number of messages sent by the notification nodes, and the total amount of outgoing data. The simulation results are depicted in Figure 5, Figure 6, Figure 7, and Figure 8.

Figure 5 and Figure 6 show the message arrival rate and the total amount of outgoing data where the node speed is twenty meters per ten seconds, respectively. Similarly, Figure 7 and Figure 8 show where the node speed is ten meters per ten seconds, respectively. Figure 5 and Figure 7 show that the message arrival rate of the GBMA is always better than that of the mobile agent within the range of the expected zone sizes in this experiment. This is because the mobile agent not reacting to its host node movements goes outside the required zone where it does not receive the notification messages. On the other hand, the GBMA migrates to receive the messages in response to the node movement. The result shows that the GBMA effective for data retrieval, relative to the conventional mobile agent.

Let us consider the results of the GBMA. When the expected zone size is large, the message arrival rate is low. A large expected zone means that the GBMA starts to migrate relatively late in response to the node movement. Therefore, the duration for which the GBMA is outside the required zone becomes long. On the contrary, when the expected zone is small, the message ar- 
rival rate is also low. A small expected zone means that the GBMA frequently migrates. Therefore, the duration for which the GBMA is migrating becomes long. By appropriately configuring the expected zone size, the message arrival rate can be increased. In Figure 5, for an appropriate expected zone size, we observe that the rate improves by as much as $32 \%$. Moreover, the results in Figure 5 and Figure 6 indicate that the optimal expected zone size will depend at least on the node speed. The optimal expected zone becomes bigger when the node speed becomes higher, because the time from when the node leaves the expected zone to when it leaves the required zone becomes shorter. The expected zone should be configured on the basis of the node speed. Figure 7 and Figure 8 show that the GBMA produces a larger number of messages than does the mobile agent. However, at the optimal expected zone size, the increment is not very large. In Figure 7, the increment with the optimal expected zone size is about only $11 \%$. From these results, with the expected zone, the start time and frequency of GBMA remigration can be easily adjusted. Moreover, appropriate adjustment of expected zone size can improve the GBMA performance.

\section{Related works}

The battery problem is a major issue in a MANET[13]. Marinescu et al. proposed a method for conserving the battery of nodes by classifying the mobile devices on the basis of the node capability and specifying the role of each node in data transmission on the basis of that classification[14]. Song et al. proposed localized algorithms for energy-efficient routing structures[16]. Gitzenis and Bambos focused on data prefetch and proposed a method for minimizing the battery consumption of a certain node by postponing data prefetching when the link quality is low, and by proactively prefetching data items when the link quality is high[15]. Subramanian et al. proposed a battery-state-aware MAC protocol[17]. These strategies focus on message routing protocol or MAC protocol, and reduce the energy consumption in each message transmissions, but do not reduce the number of messages. Therefore, when many messages are transferred at application level, with only those methods, a large amount of battery power will be consumed. Applications using directed flooding with a location information[4][5][6][9] can reduce the number of messages by limiting node forwarding of the message, but does not provide sufficient reduction in some cases which we have described. We focus on application level, and propose a location-specific data retrieval application minimizing the number of messages, using a mobile agent.

Location tracking of the mobile agent is important for message exchanges with the mobile agent. Roth and Peters proposed a scalable and secure global tracking service for mobile agents with a method similar to the global hash 
table[18]. Li and Lam proposed a location update and search algorithm for tracking mobile agents[19]. Since these works target mobile agents in an internet, they will not be directly applicable to mobile agents in a MANET whose topology dynamically changes. We restrict the mobile agent location using a geographic data and realize simple tracking of the mobile agent. Moreover, by restricting the mobile agent location, efficient messaging to the mobile agent can be feasible by directed flooding with geographic data.

\section{Conclusion}

In this paper, we proposed the Geographic Bound Mobile Agent, the required zone, and the expected zone for a data retrieval of location-specific data in a MANET. With the GBMA migrating to remain near data sources, even when an observer is far from data sources and exchanges many messages with them, data can be retrieved with a small number of messages. Simulation results show that overhead of the GBMA approach is hardly affected by the distance between the observer and the designated region, whereas overhead of the simple geocast approach increases exponentially according to it. The required zone clarifies location of the GBMA and reduces overhead of looking up the GBMA, and the expected zone easily adjusts a start timing and a frequency of GBMA migration. Simulation results show that message arrival rate in the GBMA approach with appropriate expected zone size is $32 \%$ greater than that in the conventional mobile agent approach, in the case that each node speed is about $20 \mathrm{~m} / 10 \mathrm{sec}$.

Some issues still remain unresolved. In this work, the expected zone size was static. Because the speed of each node is different and is dynamically changed, the expected zone size should be dynamically adjusted in response to the speed of the current host node, and change its shape on the basis of the direction of the node movement. Moreover, the GBMA selected a candidate node for its migration destination on the basis of only current node locations, but this is insufficient. We should consider a more sophisticated selection with more properties such as node movement speed or node movement direction. We will take up these issues in a future work.

\section{References}

[1] J. Macker and I. Chakeres, IETF working group for mobile ad-hoc networks (MANET) charter, http://www.ietf.org/html.charters/manet-charter.html, 2002.

[2] M. Andreas, L. Thomas, R. Thomas, K. Thomas and K. Holger, Design Challenges for an Integrated Disaster Management Communication and Information System, Workshop on Disaster Recovery Networks (DIREN), 2002.

[3] J.C. Navas and T. Imielinski, Geocast-geographic addressing and routing, Proceedings of International Conference on Mobile Computing and Networking (MobiCom), ACM/IEEE, 1997. 
[4] Y.B. Ko and N.H. Vaidya, Flooding-based Geocasting Protocols for Mobile Ad Hoc Networks, ACM/Baltzer Wireless Networks (WINET) journal, Vol. 7-6, pp. 471-480, 2002.

[5] I. Stojmenovic, A.P. Ruhil and D.K. Lobiyal, Voronoi Diagram and Convex Hull-Based Geocasting and Routing in Wireless Networks, Proceedings of IEEE International Symposium on Computers and Communications, pp. 51, 2003.

[6] W.H. Liao, Y.C. Tseng, K.L. Lo and J.P. Sheu, GeoGRID: A Geocasting Protocol for Mobile Ad Hoc Networks Based on GRID, Journal of Internet Technology, Vol. 1-2, pp. 23-32, 2000 .

[7] J. White, Mobile Agents White Paper, General Magic, 1996.

[8] R.S. Gray, et al., Mobile-Agent versus Client/Server Performance: Scalability in an Information-Retrieval Task, Proceedings of International Conference of Mobile Agents (MA), 2001.

[9] Y.B. Ko and N.H. Vaidya, Location-Aided Routing(LAR) in Mobile Ad hoc Networks,

[10] D.B. Johnson, D.A. Maltz, and J. Broch, DSR: The Dynamic Source Routing Protocol for Multi-Hop Wireless Ad-Hoc Networks, Ad Hoc Networking, edited by Charles E. Perkins, Chapter 5, pp. 139-172, Addison-Wesley, 2001.ACM/Baltzer Wireless Networks (WINET) journal, Vol. 6-4, pp. 307-321, 2000.

[11] R. Barr, Z.J. Haas and R. Renesse. JiST:Embedding Simulation Time into a Virtual Machine, Proceedings of EuroSim Congress on Modelling and Simulation (EuroSim), 2004.

[12] T. Camp, J. Boleng and V. Davies, A Survey of Mobility Models for Ad Hoc Network Research, Wireless Communication and Mobile Computing (WCMC): Special Issue on Mobile Ad Hoc Networking Research, Trends and Applications, Vol. 2, No. 5, pp. $483-$ $502,2002$.

[13] C.E. Jones, K.M. Sivalingam, P. Agrawal and J.C. Chen, A Survey of Energy Efficient Network Protocols for Wireless Networks, Wireless Networks, Vol. 7, No. 4, pp. 343-358, 2001 .

[14] D.C. Marinescu, G.M. Marinesxu, Y. Ji, L. Boloni and H.J. Siegel, Ad Hoc Grids: Communication and Computing in a Power Constrained Environment, Workshop on EnergyEfficient Wireless Communications and Networks (EWCN), 2003,

[15] S. Gitzenis and N. Bambos, Efficient Data Prefetching for Power-Controlled Wireless Packet Networks, Internetional Conference on Mobile and Ubiquitous Systems (MobiQuitous), 2004.

[16] W.Z. Song, Y. Wang and X.Y. Li, Localized Algorithms for Energy Efficient Topology in Wireless Ad Hoc Networks, International Symposium on Mobile Ad Hoc Networking and Computing (MobiHoc), 2004.

[17] J. Subramanian, M. Bs and S.R. Murthy, On Using Battery State for Medium Access Control in Ad hoc Wireless Networks, International Conference on Mobile Computing and Networking (MobiCom), 2004.

[18] V. Roth and J. Peters, A Scalable and Secure Global Tracking Service for Mobile Agents, Proceedings of International Conference of Mobile Agents (MA), 2001.

[19] T.Y. Li and K.Y. Lam, An Optimal Location Update and Searching Algorithm for Tracking Mobile Agent, Proceedings of International Conference on Autonomous Agents and Multiagent Systems (AAMAS), 2002. 